\title{
GPPS-CH-2020-0013
}

\section{EVALUATION OF SPALART-ALLMARAS TURBULENCE MODEL FORMS FOR A TRANSONIC AXIAL COMPRESSOR}

\author{
Xiao He*, Fanzhou Zhao and Mehdi Vahdati \\ Department of Mechanical Engineering, Imperial College London \\ London SW7 2AZ, UK
}

\begin{abstract}
A comparative study of the state-of-the-art modified Spalart-Allmaras (SA) model forms has been conducted on the transonic axial compressor NASA Rotor 67. The models studied include the SA-QCR model that improves the Boussinesq assumption, the SA-RC model that tunes turbulence production considering rotation and curvature, the SA-H model that enhances turbulence source using helicity, and the SA-PG model that boosts turbulence source using adverse pressure gradient. By comparing the results in performance characteristics and radial, circumferential and chordwise profiles, the original SA model is found to over-predict the tip blockage size and thus cause large discrepancies in the near-stall performance. The SA-QCR and SA-RC models show limited effects on the tip blockage and the near-stall performance, whereas the SA-H and SA-PG models show improved predictions, although still not near perfection. The findings suggest the requirement of a new model for better prediction of compressor tip blockage.
\end{abstract}

\section{INTRODUCTION}

The prediction accuracy on the aerodynamic stability boundary of compressors is essential to the gas turbine industry. During the past 80 years of research, several types of compressor instability have been identified, whose characteristic length ranges from the blade pitch (rotating instability) to the compressor streamwise length (surge) [1]. The multi-scale nature of compressor instability makes the application of scale-resolving simulations prohibitively expensive, because it requires a refined mesh in a large simulation domain to capture both the small scales and the large scales. Hence, Reynolds-Averaged Navier Stokes (RANS) simulation with a turbulence model will still be the workhorse for predicting compressor stability in the foreseeable future.

It is well-established that the RANS turbulence models are not accurate enough in predicting compressor near-stall flows $[1,2]$. This deficiency originates from the uncertainty involved in the development of the turbulence model. According to the level of empiricism involved in a turbulence model, the source of uncertainty can be classified into four levels [3]. The Level 1 uncertainty comes from the loss of information of fluctuating flows during the ensemble averaging process, which is fundamentally irrecoverable and sets up a ceiling of RANS models. The Level 2 uncertainty appears when a specific constitutive relation for the Reynolds stress tensor is proposed. A typical example is the Boussinesq assumption used for eddy viscosity models. The Level 3 uncertainty is generated when specific forms of transport equations are involved in the turbulence model. Finally, the Level 4 uncertainty appears when deterministic values are assigned to the model coefficients. In the previous research by the authors, the parametric uncertainty of the Spalart-Allmaras (SA) turbulence model [4] was studied in 2D canonical flows and NASA Rotor 67. It was found that the uncertainty band of the predicted stall mass flow did not envelop the measured data [5], and re-calibration of the model coefficients led to contradictory results when considering multiple flow quantities of interest [6]. Therefore, modifications at Level 4 does not appear useful for predicting compressor flows. In this work, the potential in modifying the SA model form at Level 2 and 3 is explored.

The effect of turbulence model forms on predicting detailed flow fields of compressors has been reported in several previous research. The investigated compressor flow features include but not limited to: (1) inhomogeneous jet-wake mixing; (2) corner separation and (3) tip leakage vortex. In the following literature review, case studies representing research progress on each of the three flow features are discussed 
accordingly.

The inhomogeneous jet-wake flow structure was studied on the transonic centrifugal compressor Radiver from RWTH Aachen University. Conventional linear eddy-viscosity models and non-linear Reynolds stress models predicted qualitative similar jet-wake structures with each other, but the predicted intensity and location of the jet and the wake were different from the measurements [7]. By introducing the rotation and curvature correction in the SA and SST models, the contribution of the concave/convex endwalls to the turbulence intensity can be captured by the turbulence model, and thus improve the prediction of the jet-wake structure [7-9].

The corner separation was studied on the PVD linear compressor cascade from Cambridge University. The linear eddy-viscosity models including SA, $k-\omega$ and SST were found to over-predict the separation size, but other linear models including $k-\varepsilon$ and its variants and non-linear models including $v^{2}-f$ and Reynolds stress model predicted the separation size reasonably well [10]. Therefore, modifications of the less capable models at either the transport equation level or the constitutive relation level can potentially improve their prediction in corner separation. Later studies confirmed this feasibility in the SA model: regarding the constitutive relation, replacing the linear constitutive relation by a quadratic constitutive relation achieves better results; regarding the transport equation, introducing an additional source term based on rotation and curvature, laminar-turbulent transition or helicity also improves the prediction accuracy $[11,12]$.

The tip leakage vortex was studied on a series of low-speed axial compressor from Beihang University. The trajectory of the tip leakage vortex at the design condition was predicted accurately by the SA model and the Reynolds stress model, while at the near-stall condition the SST model and the $v^{2}-f$ model shows the best accuracy. Detailed comparisons on the Reynolds stress components showed that only the Reynolds stress model predicted the Reynolds stress components with reasonable accuracy, but other models including SA, $k-\varepsilon$, SST and $v^{2}-f$ underestimated the shear stress in regions of the tip leakage vortex and the corner vortex [13]. Such findings imply that the modifications at both the transport equation and the constitutive relation levels are required in order to predict the Reynolds stress anisotropy and the turbulence intensity correctly. Numerical tests with modified transport equations of the SST model showed that an increased turbulence production improved the prediction of tip blockage [14].

In this paper, the state-of-the-art modified SA models are evaluated for a transonic fan rotor, which is of contemporary design interest but has been seldom addressed in previous works. These modified SA models include the one with a quadratic constitutive relation (SA-QCR) [15] and one with rotation and curvature correction (SA-RC) [16], both of which have been widely implemented in several computational fluid dynamics (CFD) solvers and tested in a broad range of cases (i.e., reaching model readiness rating level 3 [17]). In addition, emerging modifications using helicity (SA-H) [18] and pressure gradient (SA-PG) [19] are also evaluated since they were found useful in fan rotor stall prediction [20,21].
In the following sections, the formulations and the physical justifications of the SA model forms will be discussed first, followed by the evaluation of each SA model forms on predicting detailed flow fields of NASA Rotor 67.

\section{METHODOLOGY}

\subsection{Spalart-Allmaras Turbulence Model Forms}

2.1.1 Original Model Form The SA turbulence model [4] is based on the Boussinesq assumption in Eqn. (1):

$$
\tau_{i j}=2 \mu_{t}\left(S_{i j}-\frac{1}{3} \frac{\partial u_{k}}{\partial x_{k}} \delta_{i j}\right)
$$

where the turbulent shear stress $\tau_{i j}$ is linearly corelated with the mean strain rate $S_{i j}$, and the eddy viscosity $\mu_{t}$ needs to be calculated by an eddy viscosity turbulence model. The transport equation of the SA turbulence model is shown in Eqn. (2):

$$
\begin{aligned}
& \frac{\partial \hat{v}}{\partial t}+u_{j} \frac{\partial \hat{v}}{\partial x_{j}}=\underbrace{\left[c_{b 1}\left(1-f_{t 2}\right) \hat{S} \hat{v}\right]}_{\text {production }}-\left[c_{w 1} f_{w}-\frac{c_{b 1}}{\kappa^{2}} f_{t 2}\right]\left(\frac{\hat{v}}{d}\right)^{2} \\
& \text { destruction } \\
& \underbrace{+\frac{1}{\sigma}\left[\frac{\partial}{\partial x_{j}}\left((v+\hat{v}) \frac{\partial \hat{v}}{\partial x_{j}}\right)+c_{b 2} \frac{\partial \hat{v}}{\partial x_{i}} \frac{\partial \hat{v}}{\partial x_{i}}\right]}_{\text {diffusion }}
\end{aligned}
$$

with additional functions calculated in the following:

$$
\begin{aligned}
\mu_{t} & =\rho \hat{v} f_{v 1} & \hat{S} & =\Omega+\frac{v}{\kappa^{2} d^{2}} \chi f_{v 2} \\
c_{w 1} & =\frac{c_{b 1}}{\kappa^{2}}+\frac{1+c_{b 2}}{\sigma} & f_{v 1} & =\frac{\chi^{3}}{\chi^{3}+c_{v 1}^{3}} \\
f_{v 2} & =1-\frac{\chi}{1+\chi f_{v 1}} & f_{w} & =g\left[\frac{1+c_{w 3}^{6}}{g^{6}+c_{w 3}^{6}}\right]^{1 / 6} \\
g & =r+c_{w 2}\left(r^{6}-r\right) & r & =\min \left[\frac{\hat{v}}{\hat{S} \kappa^{2} d^{2}}, 10\right] \\
f_{t 2} & =c_{t 3} \exp \left(-c_{t 4} \chi^{2}\right) & &
\end{aligned}
$$

where $\rho$ is density, $d$ is wall distance, and $\sigma=0.667, \kappa=0.41$, $c_{v 1}=7.1, c_{b 1}=0.1355, c_{b 2}=0.622, c_{w 2}=0.3, c_{w 3}=2.0$, $c_{t 3}=1.2$ and $c_{t 4}=0.5$ are model coefficients. To improve the numerical stability, $\hat{S}$ is limited to be greater than zero.

2.1.2 Model Modification at Level 2 It is well established that the linear correlation of Boussinesq assumption in Eqn. (1) is deficient in 3D and separated flows [22]. To modify the Boussinesq assumption, Spalart [15] proposed an additional quadratic term as described in Eqn. (4):

$$
\tau_{Q C R, i j}=\tau_{i j}-2 c_{c r 1} \mu_{t}\left[O_{i k} S_{j k}+O_{j k} S_{i k}\right]
$$


where the coefficient $c_{c r 1}=0.3$ was calibrated in the outer region of a simple boundary layer, and the additional terms are calculated as:

$$
\begin{aligned}
O_{i j} & =2 W_{i j} / \sqrt{\frac{\partial u_{m}}{\partial x_{n}} \frac{\partial u_{m}}{\partial x_{n}}} \quad W_{i j}=\frac{1}{2}\left(\frac{\partial u_{i}}{\partial x_{j}}-\frac{\partial u_{j}}{\partial x_{i}}\right) \\
S_{i j} & =\frac{1}{2}\left(\frac{\partial u_{i}}{\partial x_{j}}+\frac{\partial u_{j}}{\partial x_{i}}\right)
\end{aligned}
$$

The above quadratic constitutive relation applied to the SA model is referred to as the SA-QCR model. It should be noted that the trace of the additional Reynolds stress tensor is zero. Thus, the quadratic term does not change the turbulent kinetic energy or the total Reynolds stress budget. It only adjusts the anisotropy of the Reynolds stress tensor or redistributes the Reynolds stress components.

2.1.3 Model Modification at Level 3 The majority modifications of the SA transport equation involve multiplying the source terms by an empirical function of physical terms. In this work, the modification with respect to rotation and curvature [16], helicity [18] and pressure gradient [19] are considered, and these models will be referred to as SA-RC, SA-H and SA-PG respectively. Note that the implementation of these models in this work may differ from the original ones, and these differences are explained in detail in the following.

The SA-RC model considers that a strong shear enhances turbulence intensity whereas a strong rotation reduces it. In addition, the effect of streamline curvature leads to an effective rotation rate, which can be treated in a similar fashion to the rotation effect. The above features are achieved by replacing the SA production term with $c_{b 1}\left(f_{r 1}-f_{t 2}\right) \hat{S}$, where the $f_{r 1}$ function is defined in Eqn.(6):

$$
f_{r 1}\left(r^{*}, \hat{r}\right)=\left(1+c_{r 1}\right) \frac{2 r^{*}}{1+r^{*}}\left[1-c_{r 3} \tan ^{-1}\left(c_{r 2} \hat{r}\right)\right]-c_{r 1}
$$

In this equation, the coefficient $c_{r 1}=1.0$ was calibrated in wingtip flows, the coefficients $c_{r 2}=12$ and $c_{r 3}=1.0$ were calibrated in curved and rotating boundary layers, and additional terms are calculated as:

$$
\begin{aligned}
r^{*} & =S / \omega \\
\hat{r} & =\frac{8 \omega_{i k} S_{j k}}{\left(S^{2}+\omega^{2}\right)^{2}}\left(\frac{D S_{i j}}{D t}+\left(\varepsilon_{i m n} S_{j n}+\varepsilon_{j m n} S_{i n}\right) \Omega_{m}^{\prime}\right) \\
S_{i j} & =\frac{1}{2}\left(\frac{\partial u_{i}}{\partial x_{j}}+\frac{\partial u_{j}}{\partial x_{i}}\right) \\
\omega_{i j} & =\frac{1}{2}\left[\left(\frac{\partial u_{i}}{\partial x_{j}}-\frac{\partial u_{j}}{\partial x_{i}}\right)+2 \varepsilon_{m j i} \Omega_{m}^{\prime}\right]
\end{aligned}
$$

It should be noted that the original definition of $r^{*}$ has a singularity at $\omega=0$. To avoid the singularity, a reference strain rate $\varepsilon=u_{r e f} / l_{r e f}$ is usually added to both the denominator and the numerator of $r^{*}$, where $u_{r e f}$ and $l_{r e f}$ are characteristic velocity and length scale of the case. For pure shear flows, the turbulence intensity is enhanced since $r^{*} \rightarrow+\infty, \hat{r}=0$ and thus $f_{r 1}=2+c_{r 1}>1$. For pure rotation flows, the turbulence intensity is reduced because $r^{*}=0, \hat{r}=0$ and hence $f_{r 1}=-c_{r 1}<1$.

The SA-H model tries to account for the turbulence energy backscatter using the velocity helicity. It multiplies the vorticity $\Omega$ in the modified strain rate term $\hat{S}$ by an empirical function $\beta_{h}(h)$ :

$$
\begin{aligned}
\beta_{h}(h) & =1+c_{h 1} \tanh \left(c_{h 2} h^{c_{h 3}}\right) \\
h & =\frac{\left|\Omega_{i} u_{i}\right|}{\Omega U}
\end{aligned}
$$

In Eqn. (8), the form $c_{h 1} \tanh \left(c_{h 2} h^{c_{h 3}}\right)$ is used instead of the original proposal $c_{h 1} h^{c h 2}$ so that an additional degree of freedom is available for calibration. For consistency with the original $\beta_{h}(h)$ function, the coefficients are calibrated as $c_{h 1}=$ $0.71, c_{h 2}=2.0$ and $c_{h 3}=1.0$. An increase in helicity leads to a higher modified strain rate, which enhances turbulence intensity via both the production and the destruction terms in Eqn.(2). Since helicity in 2D flows is always zero, the modification has no effect for these flow cases. In addition, the velocity helicity is not Galilean-invariant, and it is by default calculated in the relative frame in this work. The absence of Galilean-invariance will cause discontinuity in eddy viscosity across the rotor-stator interface of a compressor stage, especially when 3D stall cells develop across the interface.

The SA-PG model considers a possible correlation between the pressure gradient and the turbulence intensity. Similar to the SA-H model, the vorticity $\Omega$ in the modified strain rate $\hat{S}$ is multiplied by an empirical function $\beta_{p}(\hat{p})$ :

$$
\begin{aligned}
\beta_{p}(\hat{p}) & = \begin{cases}1+c_{p 1} \tanh \left(c_{p 2} \hat{p}^{c_{p 3}}\right), & \text { if } \frac{\partial p}{\partial x_{i}} u_{i} \geq 0 \\
1, & \text { otherwise }\end{cases} \\
\hat{p} & =10^{6} \cdot \sqrt{\frac{\partial p}{\partial x_{i}} \frac{\partial p}{\partial x_{i}}} / \frac{\rho^{2} U^{3}}{\mu}
\end{aligned}
$$

Compared to the original proposal [19], the form $c_{p 1} \tanh \left(c_{p 2} \hat{p}^{c_{p 3}}\right)$ is adopted instead of the original proposal $c_{p 1} \hat{p}^{c_{p 3}} /\left(c_{p 2}+\hat{p}^{c_{p 3}}\right)$ due to better interpretations of the model coefficients. For consistency with the original $\beta_{p}(\hat{p})$ function, the coefficients are calibrated as $c_{p 1}=0.80, c_{p 2}=0.05$, $c_{p 3}=3.0$. Another difference compared to the original model is that the $\beta_{p}$ term only switches on when adverse pressure gradient is detected. This is physically justified as only adverse pressure gradient increases the turbulence intensity whereas favorable pressure gradient reduces it. It should be noted that the model is not Galilean-invariant either since the velocity vector involved in Eqn. (9) is subjected to the reference frame. The relative velocity is used in this work by default. Moreover, it is argued that the pressure gradient term is not a valid entry for a general purpose turbulence model, because the pressure gradient does not occur in the vorticity 


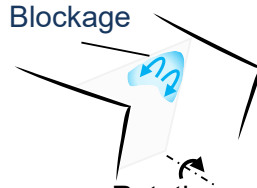

Rotation
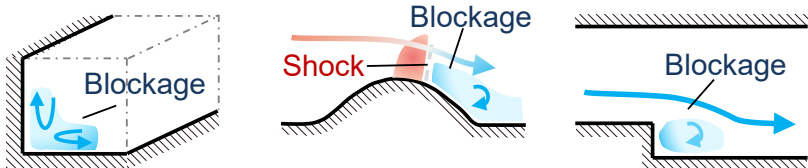

Figure 1: Illustration of compressor tip flow features and selected canonical flow cases for verification

equation of incompressible flows [23]. The SA-PG model did show worse results than the original SA model in the axisymmetric bump and the backward-facing step [6]. Thus, this model may only improve predictions on a specific type of flow.

\subsection{Flow Solver, Validation and Verification}

This study is based on a high-order implicit in-house flow solver that has hybrid RANS/LES capabilities. It uses a node-centered finite volume scheme with an edge-based data structure, and it is compatible with structured/unstructured hybrid grids. The numerical fluxes are solved by the Jameson-Schmidt-Turkel scheme, and the artificial viscosity is constructed with a hybrid low-dissipation scheme based on Roe's matrix. The steady RANS mode of the solver with second-order spatial accuracy is used throughout the paper.

In the first place, the in-house code was verified against established CFD codes such as CFL3D, FUN3D and OVERFLOW $[17,24]$. The verification cases include a square duct, an axisymmetric bump and a backward-facing step, as illustrated in Fig.1. These canonical flows resembles part of the compressor tip flow features, such as secondary flow, shock-boundary layer interaction and geometry-induced separation. In order to make a direct comparison against these CFD codes, the computations were performed on the same structured grid and the same boundary conditions as used by the other codes. Details of the grid and the boundary conditions of the canonical flows can be found in the NASA Turbulence Modeling Resource website [17].

In Fig.2(a), the predicted skin friction of the square duct is compared to that from literature [17]. Since the SA and SA-QCR results obtained in this work overlap with the results obtained by using other validated CFD codes, the implementation of the SA and SA-QCR models is verified. In Fig.2(b) and (c), the wall pressure coefficient of the axisymmetric bump and the skin friction of the backward-facing step are shown. Again, it is verified that the results obtained by the SA and SA-RC models in this work are almost identical to that from other solvers $[17,24]$.

\subsection{Case Description}

The transonic fan rotor studied in this work is NASA Rotor 67, which is a high-speed first-stage rotor of a two-stage fan. Detailed geometry data and extensive measurement data of this case can be found in Strazisar et al. [25], and more recent performance data can be found in Grosvenor [26] and Fidalgo et al. [27].

The quantities of interest include characteristics of total pressure ratio and isentropic efficiency, radial profiles at the rotor exit, and circumferential and chordwise profiles at $90 \%$ span. The corresponding measurement planes are illustrated in Fig.3. The measured performance characteristics were based on the pressure and temperature probe data at the rotor inlet and exit, and the mass flow was measured at the downstream orifice. The pressure was work-weighted averaged over each measurement plane, while the temperature was mass-weighted averaged. Since the difference between the two averaging methods is usually small [28] (e.g., $\pm 0.01 \%$ in total pressure ratio in the current case), the mass-weighted average method is practiced throughout the paper. Uncertainties of the total pressure ratio and the isentropic efficiency are calculated from the pressure probe uncertainty of $1 \mathrm{kPa}$ and the temperature probe uncertainty of $0.6 \mathrm{~K}$. The radial profiles of total pressure, total temperature and absolute flow angle were also based on the probe data, and they were circumferentially mass-weighted averaged. The circumferential and chordwise profiles of relative Mach number and relative flow angle were based on the laser anemometer measurement. The raw data were ensemble-averaged over 50 rotor passage. In the following analysis, all the presented CFD data are obtained and post-processed in the same way as the experiment.

The computational model of NASA Rotor 67 is illustrated in Fig.4, which consists of an inlet duct, a rotor passage, an exit duct and a nozzle. At the inlet, a uniform distribution of total pressure and total temperature is set at the standard sea-level condition, the velocity vector is set in axial direction, and the eddy viscosity is set as $v_{t}=10^{-5} \mathrm{~m}^{2} / \mathrm{s}$ following the recommended value range of $v_{t} / v$ between 0.2 and 1.3 [29]. At the exit, a low static pressure is set to choke the nozzle, and the operating condition of the compressor can be controlled by changing the nozzle area [30]. The mixed-element unstructured mesh used in this case has 1.4 million mesh points in the rotor domain and 0.8 million mesh points in the piping system. The average $y^{+}$value of the near-wall mesh is kept below 3 , and the boundary condition at the viscous wall is calculated by the standard wall function [31]. A tip gap size of $0.61 \mathrm{~mm}$ and a hub fillet radius of $1.78 \mathrm{~mm}$ have been meshed with 20 and 16 spanwise layers, respectively. When the residual of density and the operating mass flow do not drop with further iterations, the simulation is considered converged.

In addition to the errors in the turbulence model, there are other error sources affect the prediction accuracy as discussed in the following.

1. Discretization error. A mesh independence study has been conducted using the original SA model. It is found that the change of total pressure ratio between the peak efficiency (PE) condition (i.e., $m / m_{c}=0.989 \pm 0.004$ ) and 

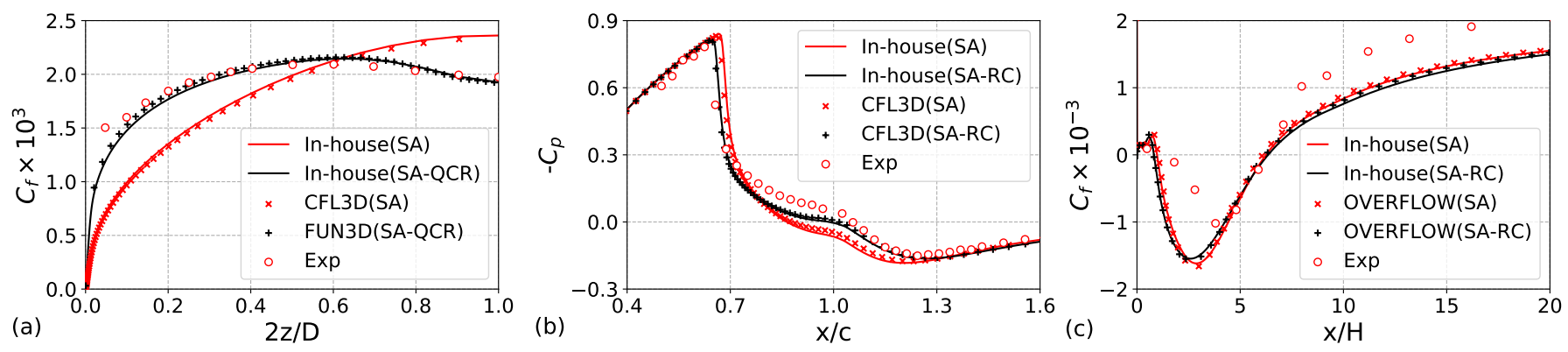

Figure 2: Validation and verification: (a) skin friction at $x=50 \mathrm{D}$ of the square duct; (b) wall pressure coefficient of the axisymmetric bump; (c) skin friction of the backward-facing step

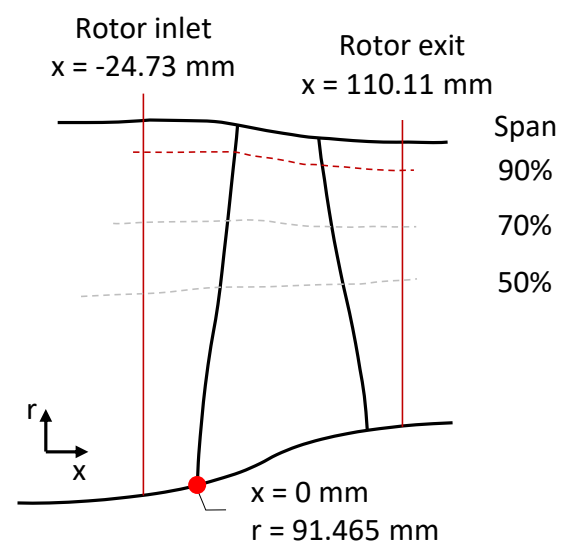

Figure 3: Measurement planes of NASA Rotor 67

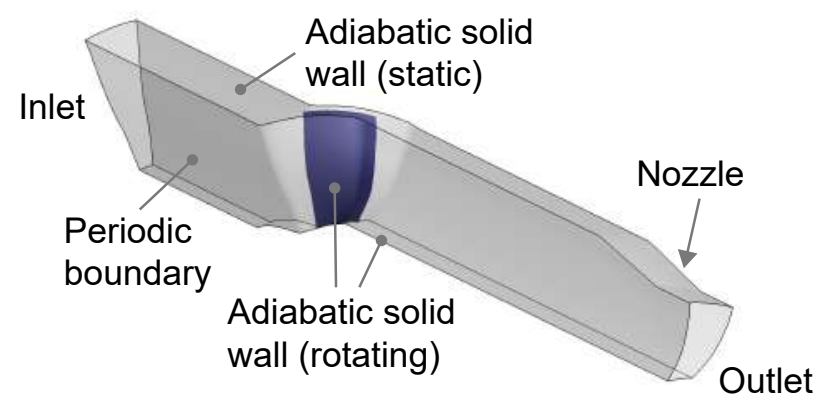

Figure 4: Flow domain and boundary conditions for NASA Rotor 67

the near stall (NS) condition (i.e., $m / m_{c}=0.924 \pm 0.004$ ) is within $\pm 0.1 \%$ when the mesh is refined further than 1.4 million mesh points. This is consistent with the previous mesh independence studies using different second-order accurate solvers $[26,32]$.

2. Uncertainty of inlet boundary conditions. Due to a lack of knowledge about the inlet piping system of the test rig, rotor inlet profiles of flow quantities from CFD may differ slightly from those measured in the experiment. In the experiment, the rotor inlet profiles of total pressure, total temperature and velocity were provided only at the PE and the NS conditions [25]. The freestream viscosity ratio $v_{t} / v$ at the rotor inlet is calculated around 200, which is based on the measured turbulence intensity of $1.5 \%$ and the estimated turbulence length scale of $22 \%$ of the boundary layer thickness [33]. Additional numerical tests have been performed to match up with the prescribed rotor inlet conditions. Results indicate a $\pm 0.5 \%$ variation in total pressure ratio due to the uncertainty of inlet boundary conditions.

3. Steady-state assumption. The internal flow of compressor is by nature unsteady, but it is often assumed that the steady simulation results are reasonably close to the time-averaged unsteady solution. An unsteady simulation has been performed at the NS condition using the same single-passage mesh and the original SA model. The time period for each rotor revolution is discretized by 660 time steps. Within each time step, 30 Newton iterations are performed so that the residual of density drops by two orders of magnitude at least. The simulation starts from the converged steady solutions and runs for over 20 rotor revolutions. Instantaneous solutions of the last 5 rotor revolutions are used for time-averaging. Results show that the error in total pressure ratio due to the steady-state assumption is within $\pm 0.1 \%$.

\section{Results}

\subsection{Performance Characteristics}

The quantities of interest in the NASA Rotor 67 case include the performance characteristics and the stall boundary at the design speed. These quantities are shown in Fig.5.

The pressure ratio characteristics from different turbulence models are presented in Fig.5(a). In general, the shape of the characteristic curves predicted by the SA, SA-QCR and SA-RC models are similar to each other. However, all the three models predict stall prematurely compared with the measurement and the predictions from SA-PG and SA-H. Moreover, the SA-H model predicts higher pressure ratio than all the other models for all mass flows. At the PE condition of the experiment, the pressure ratio is predicted accurately by the SA-PG model, but it is over-predicted by the SA-H model and under-predicted by the SA, SA-QCR and SA-RC models. The variation range of the predicted pressure ratio is about $3.0 \%$ of the measured value at PE. At the NS condition of the experiment, the SA-H and 

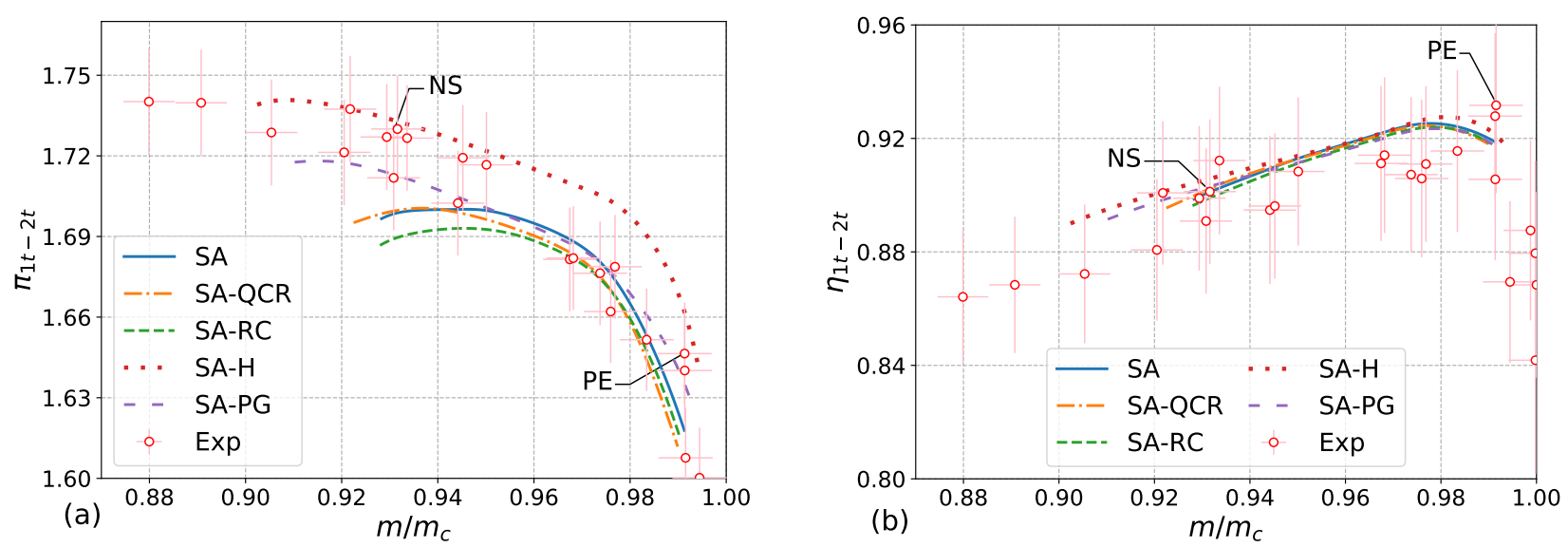

Figure 5: Performance characteristics of NASA Rotor 67: (a) pressure ratio and (b) isentropic efficiency

the SA-PG model match well with the measured data, but the SA, SA-QCR and SA-RC models under-predict the pressure ratio. The variation range of the predicted pressure ratio is about $2.4 \%$ of the measured value at NS.

The isentropic efficiency characteristics are compared in Fig.5(b). Because of the large measurement uncertainty in the experiment, all the predicted characteristic curves fall within the measured data and are considered reasonably accurate. Besides, the characteristic curves of all the models are similar to each other in terms of the overall shape and the mass flow of the predicted PE point. The largest difference occurs near stall, where the variation range of efficiency is about $0.5 \%$ in absolute value.

\subsection{Tip Blockage}

The NASA Rotor 67 presents a spike-type rotating stall pattern [32], which is triggered by the formation of a tip blockage cell that leads to the spillage of the tip leakage flow at the leading edge (LE) and the reversal of the tip leakage flow at the trailing edge (TE) [34]. In Fig.6(a), the circumferentially averaged axial velocity contour is presented where the tip blockage cell is enveloped by the isoline of $u=0$. At the last converged point predicted by the SA model (i.e., $m / m_{c}=0.928$ ), the tip blockage cell extends from the LE to the TE, indicating the source of blockage comes from both the spillage near the LE and the tip corner separation near the TE. At the last converged point predicted by the SA-H model (i.e., $m / m_{c}=0.902$ ), the tip blockage cell only reaches the LE but not the TE. This scenario often occurs in cases where the chordwise distribution of leakage axial momentum is skewed towards the TE, and the casing corner separation is thus suppressed by the tip leakage flow [35]. For the SA-H model, the decreased blockage size is achieved by enhancing the turbulent mixing between the corner separation region and the main stream; this will be shown later in section 3.4.

The development of the tip blockage cell can be traced by its start and end locations $x_{b, s}$ and $x_{b, e}$, and the size of tip blockage cell can be measured by its axial length $L_{b}=$ $x_{b, e}-x_{b, s}$. The definitions of these variables are illustrated in Fig.6(a). In Fig.6(b), $x_{b, s}$ and $x_{b, e}$ are plotted against the mass flow rate. When operating near the choke condition, the tip blockage cell is partially outside the blade passage and its size is small. As the compressor is throttled towards stall, the tip blockage cell moves upstream in the blade passage and extends its size towards both the LE and the TE. The turbulence model has a more significant effect on $x_{b, e}$ than $x_{b, s}$, indicating the reversal of the tip leakage flow near TE is more susceptible than the spillage near LE. In Fig.6(c), $L_{b}$ is plotted against the mass flow rate. It is illustrated that the $\mathrm{SA}-\mathrm{H}$ model reduces the tip blockage size over the full speed line, which explains its over-prediction in the pressure ratio near $\mathrm{PE}$ as shown in Fig.5(a). In contrast, the SA-PG and the SA-QCR model only reduces the tip blockage when approaching stall, which are favored for better prediction accuracy. No major difference in tip blockage prediction between the original SA model and the SA-RC model is found.

To check the effect of tip blockage on the downstream flow quantities, the circumferentially averaged radial profiles of total pressure, total temperature and absolute flow angle are presented in Fig.7. Since both the total temperature and the absolute flow angle are positively correlated with the compressor work input, they are discussed together. For the measured PE condition shown in Fig.7(a), the original SA model predicts slightly lower values of total pressure ratio and work input near the tip region, and the modified model SA-PG improves the overall prediction accuracy the most. The SA-H model predicts higher pressure ratio and work input at almost the whole span, which is consistent with the observation of an under-predicted tip blockage size. The SA-QCR and the SA-RC models predict lower near-tip pressure ratio and work input. For the measured NS condition shown in Fig.7(b), pressure ratio and work input values from the SA, SA-QCR and SA-RC models are significantly under-predicted near the tip region, indicating an over-predicted tip blockage size. In contrast, both the SA-H and the SA-PG models improve the prediction, and the SA-H model shows the closest agreement with measurements. These observations are consistent with that in the performance characteristics shown in Fig.5(a).

To investigate the near-tip flow fields further, the circumferential profiles of relative Mach number and relative flow angle at $90 \%$ span of the rotor exit are presented in Fig.8. 

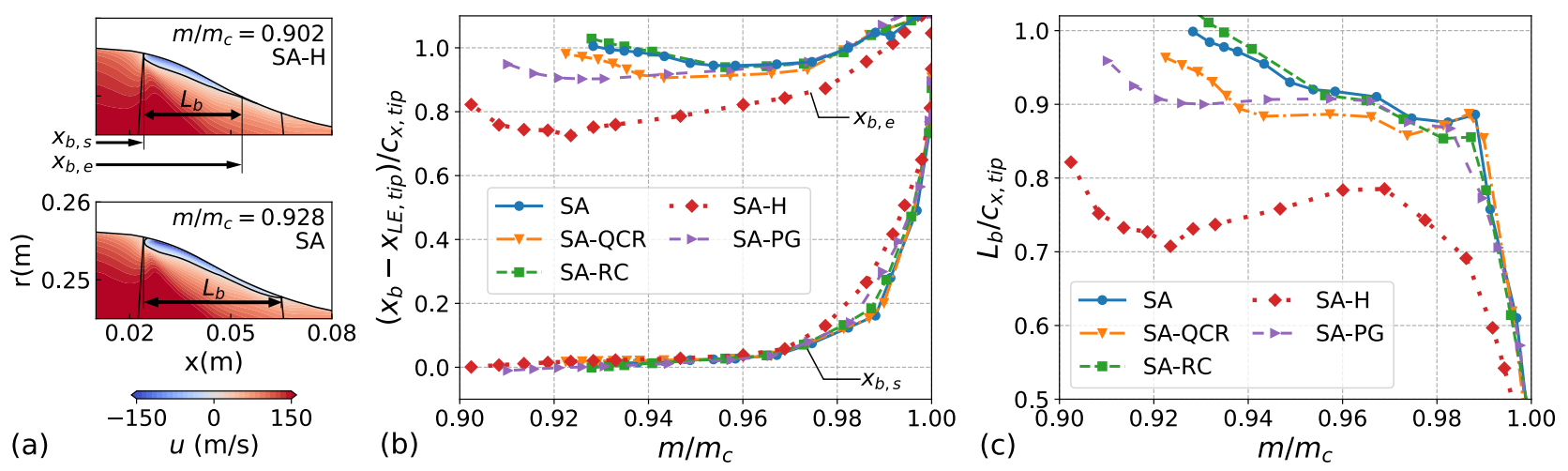

Figure 6: Blockage size of NASA Rotor 67: (a) contours of circumferentially mass-weighted averaged axial velocity; (b) start and end locations of blockage versus mass flow rate; (c) blockage size versus mass flow rate
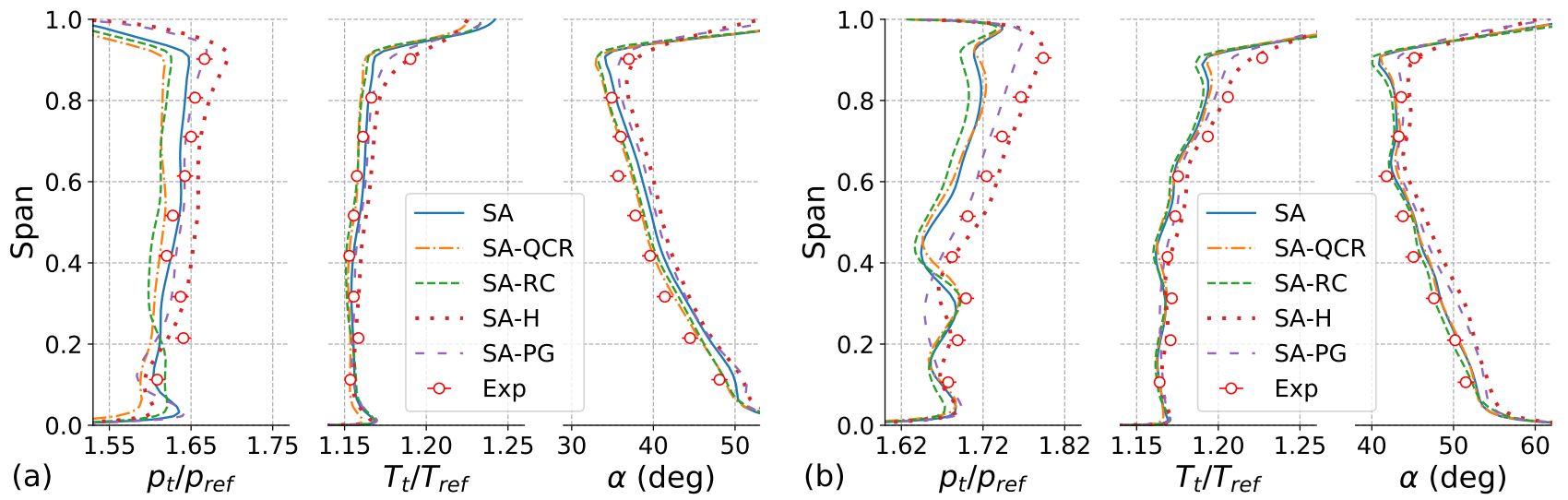

Figure 7: Circumferentially mass-weighted averaged radial profiles at the exit of NASA Rotor 67: $\left(\right.$ a) peak efficiency $\left(m / m_{c}=\right.$ $0.989 \pm 0.004)$; (b) near stall $\left(m / m_{c}=0.924 \pm 0.004\right)$

In these plots, the circumferential coordinates are normalized by the blade pitch, and the location at $100 \%$ pitch corresponds to the trailing edge of the blade. At the trailing edge location, a wake can be observed featured by a local minimum of relative Mach number and a local maximum of relative flow angle. As the measurement plane is immediately downstream of the blockage region, the change of streamline curvature around the blockage cell results in a local reduction in relative flow angle near the suction surface between $100 \%$ and $110 \%$ pitch. For the measured PE condition shown in Fig.8(a), the SA, SA-QCR and SA-RC models predict qualitatively similar results to each other, and their predictions show adequate agreement with measurements. The SA-H and SA-PG models predict lower relative Mach number in the whole pitch and a higher relative flow angle between $100 \%$ and $110 \%$ pitch, indicating these models over-suppress the tip blockage size. For the measured NS condition presented in Fig.8(b), SA-H and SA-PG models show better agreement with the experiment than the other models. These results further demonstrate that the SA-H and SA-PG models help reduce the tip blockage size, while the SA-QCR and SA-RC models have negligible effects.

\subsection{Shock Structure}

Shock and its interactions with the boundary layer and the tip leakage flow are the major source of the tip blockage. In Fig.9, the chordwise profile of relative Mach number at $90 \%$ span and mid-passage is presented to check the shock structure.

At the PE condition, the passage shock predicted by the SA-H and SA-PG models is significantly stronger and located more downstream. Similar observations can be made for the SA-QCR and SA-RC models but with a less extent. The variation of the pre-shock Mach number is about 0.1 , and that of the shock front location is about $8 \%$ of the chord length. No difference among the turbulence models can be observed in predicting the bow shock. It is reminded that the comparison is made at the same normalized mass flow of the measured PE point, and the predicted PE points have a smaller mass flow than the measured one. Therefore, the large uncertainty in the experiment presented in Fig.5(b) is the main cause of the inconsistency in the shock structure at PE condition.

At the NS condition, it is demonstrated that the turbulence model has a limited effect on the shock location, but it can change the shock strength and thus the shock-induced separation. This can be seen from the distribution of relative Mach number downstream the shock. Compared to the experiment, results obtained by the SA-H and SA-PG model 

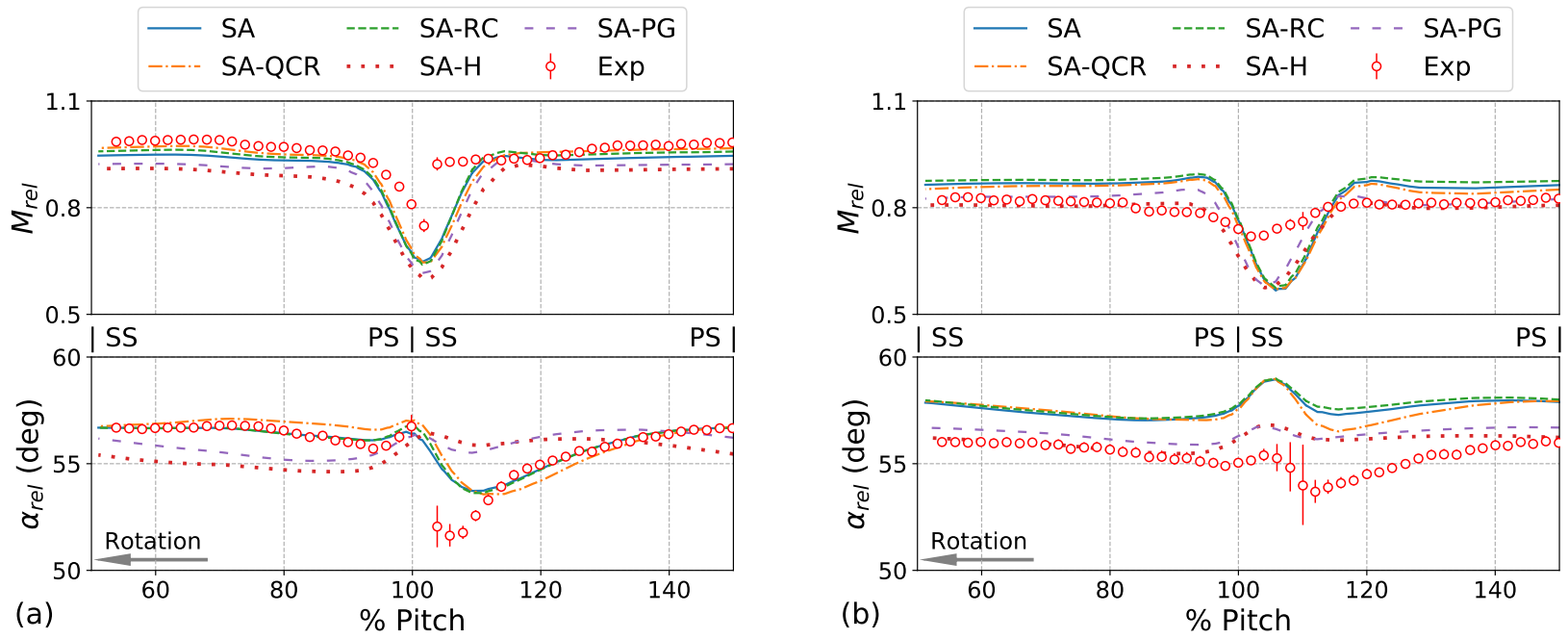

Figure 8: Circumferential profiles at $90 \%$ span and 24\% chord downstream the trailing edge of NASA Rotor 67: (a) peak efficiency $\left(m / m_{c}=0.989 \pm 0.004\right)$; (b) near stall $\left(m / m_{c}=0.924 \pm 0.004\right)$

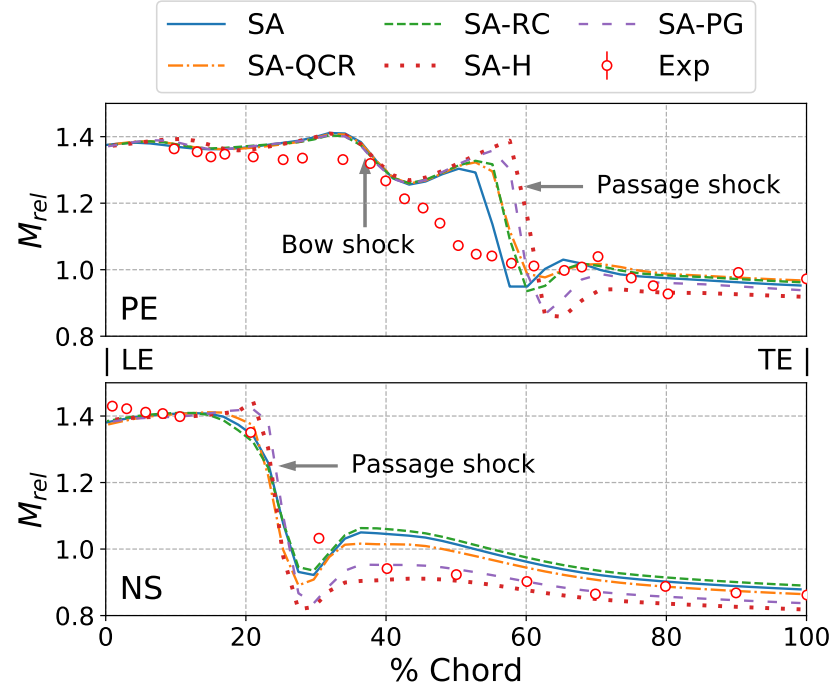

Figure 9: Chordwise profile of realtive Mach number at $90 \%$ span and $50 \%$ pitch of NASA Rotor 67

match with the measurement the best.

\subsection{Eddy Viscosity Field}

Finally, the eddy viscosity differences between the modified and the original SA model (i.e., $\left.\Delta v_{t}=v_{t, \text { mod }}-v_{t}\right)$ ) are examined in Fig.10. The eddy viscosity field is determined by both the turbulence model and the primary flow fields of velocity, pressure and temperature. For a fair comparison between different turbulence models, the primary solutions are frozen at the converged solution from the modified SA model, and the eddy viscosity $v_{t}$ and $v_{t, \text { mod }}$ are obtained by only solving the transport equation of the turbulence model without updating the primary flow fields. To better relate the change of eddy viscosity with the flow physics, the relative Mach number contours with relative streamlines near the blade tip are illustrated in Fig.11. The main features related to shock and tip leakage flow are pointed out by arrows.

In general, the SA-H model has the largest influence on eddy viscosity, while the effects of the SA-PG model and the SA-RC model rank the second and the third.

At the PE condition, the SA-RC model mainly reduces eddy viscosity in the wake region where the local rotation effect is strong, but little effect can be observed in the blade passage. The SA-PG model increases the eddy viscosity in the rear part of the blade passage corresponding to the location of the shock-induced separation. The SA-H model boosts eddy viscosity near the outer edge of the casing boundary layer. This region has a large relative helicity because the local vorticity generated by the relative motion of the casing wall is aligned with the local velocity vector. Besides, the eddy viscosity is further enhanced near the shock-enhanced tip leakage flow region where both the relative helicity and the vorticity magnitude are large.

At the NS condition, the SA-RC model reduces the eddy viscosity slightly in the trajectory of tip leakage vortex, but increases the eddy viscosity in the other parts of tip region where the effect of shear between the main flow and the tip leakage flow is more significant than the effect of rotation. The SA-PG model still enhances the eddy viscosity in the vicinity of the passage shock, but its effect at the NS condition is larger than the PE condition due to the increased magnitude of the adverse pressure gradient. The results of the SA-H model show a similar level of increment in eddy viscosity to those at the PE condition, but the affected region moves more upstream with the trajectory of the shock-induced tip leakage vortex.

\section{CONCLUSIONS AND FUTURE WORKS}

In this paper, the state-of-the-art modified versions of the Spalart-Allmaras turbulence model have been evaluated in compressor tip flow features. Several conclusions can be drawn as follows.

The original SA model is deficient in predicting the 

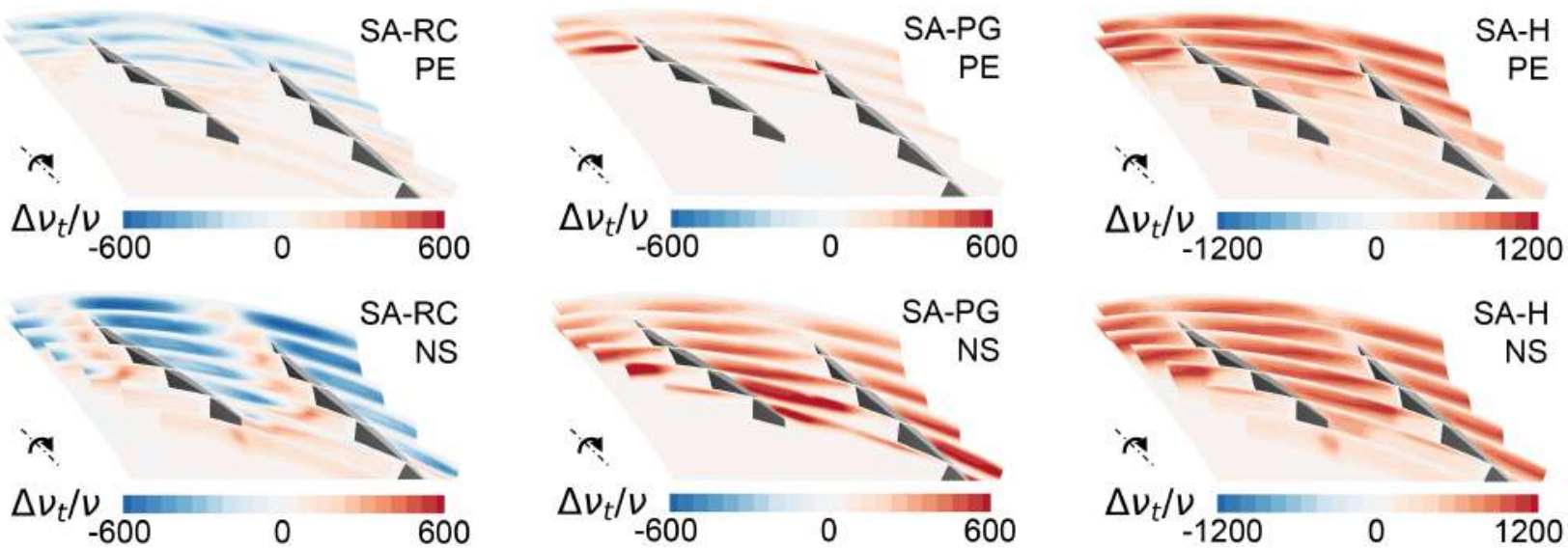

Figure 10: Contours of eddy viscosity difference between the modified SA models and the original SA model near the tip region of the NASA Rotor 67 case
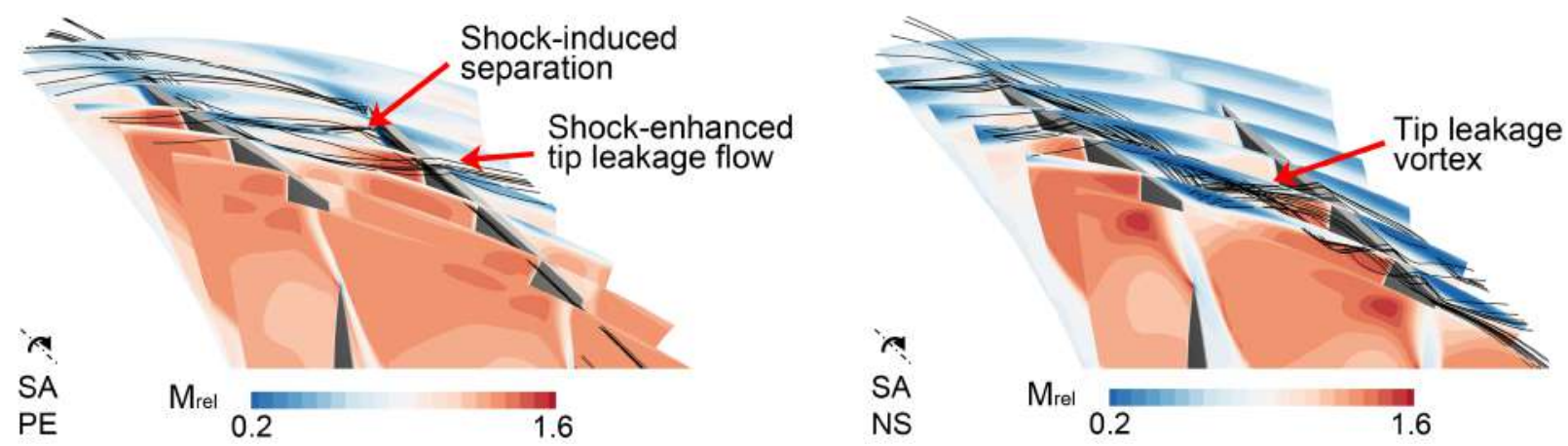

Figure 11: Contours of relative Mach number superimposed with relative streamlines near the tip region of the NASA Rotor 67 case

near stall flow of NASA Rotor 67 in terms of performance characteristics and flow quantity profiles in both the radial and the circumferential direction. The differences between the simulation and the measurement results indicate an over-prediction in the tip blockage size by the SA model.

The SA-QCR model slightly improves the prediction accuracy in terms of the blockage size, while the SA-RC model slightly worsens it. Their effects on the compressor performance characteristics are limited. Therefore, the SA-QCR and the SA-RC models are not recommended over the original SA model for compressor applications, despite their success in canonical flows.

The SA-H and SA-PG models show the best accuracy among all the models studied in predicting near-stall flows, although they are still far from perfection. The SA-H model reduces the tip blockage size at the whole speed line, which improves the prediction near stall but leads to worse prediction near peak efficiency. The SA-PG model decreases the tip blockage size depending on the adverse pressure gradient level of the operating condition, which improves the prediction at both peak efficiency and near stall conditions. However, the SA-PG model has detrimental effects on some academic flows.

For future research, a new model incorporating features of adverse pressure gradient and 3D helical flows is recommended to further improve the prediction of compressor tip blockage.

\section{ACKNOWLEDGMENT}

Xiao He greatly acknowledges the Imperial College President PhD Scholarship for funding this research.

\section{NOMENCLATURE}

$c \quad$ chord length $(m)$

$C_{p} \quad$ pressure coefficient (-)

$D \quad$ duct width $(m)$

$H \quad$ step height $(m)$

$L_{b} \quad$ blockage size $(m)$

M absolute Mach number (-)

$m \quad$ mass flow rate $(\mathrm{kg} / \mathrm{s})$

$m_{c} \quad$ choke mass flow rate $(\mathrm{kg} / \mathrm{s})$

$\mathrm{M}_{\text {rel }} \quad$ relative Mach number (-)

$p_{t} \quad$ total pressure $(\mathrm{Pa})$

Re Reynolds number (-)

$T_{t} \quad$ total temperature (K)

$\alpha \quad$ absolute flow angle (deg)

$\alpha_{\text {rel }}$ relative flow angle (deg)

$\eta \quad$ isentropic efficiency (-)

$v_{t} \quad$ kinetic eddy viscosity $\left(\mathrm{m}^{2} / \mathrm{s}\right)$

$v \quad$ kinetic molecular viscosity $\left(\mathrm{m}^{2} / \mathrm{s}\right)$

$\pi \quad$ pressure ratio (-)

$\tau_{i j} \quad$ Reynolds stress tensor $(\mathrm{Pa})$ 


\section{REFERENCES}

[1] Day, I. J., 2016. "Stall, surge, and 75 years of research". ASME J. Turbomach., 138(1), p. 011001.

[2] Denton, J. D., 2010. "Some limitations of turbomachinery CFD". ASME Paper No. GT2010-22540.

[3] Duraisamy, K., Iaccarino, G., and Xiao, H., 2019. "Turbulence modeling in the age of data". Annu. Rev. Fluid Mech., 51, pp. 357-377.

[4] Spalart, P., and Allmaras, S., 1994. "A one-equation turbulence model for aerodynamic flows". Rech. Aerospatiale, 1, pp. 5-21.

[5] He, X., Zhao, F., and Vahdati, M., 2020. "Uncertainty quantification of Spalart-Allmaras turbulence model coefficients for compressor stall". ASME Paper No. GT2020-15014.

[6] He, X., Zhao, F., and Vahdati, M., 2020. "Uncertainty quantification of Spalart-Allmaras turbulence model coefficients for simplified compressor flow features". ASME J. Fluids Eng., 142(9), p. 091501.

[7] Gibson, L., Galloway, L., Spence, S., and Kingdom, U., 2017. "Assessment of turbulence model predictions for a centrifugal compressor simulation". J. Glob. Power Propuls. Soc., 1, pp. 142-156.

[8] Dufour, G., Cazalbou, J.-B., Carbonneau, X., and Chassaing, P., 2008. "Assessing rotation/curvature corrections to eddy-viscosity models in the calculations of centrifugal-compressor flows". ASME J. Fluids Eng., 130(9), p. 091401.

[9] Smirnov, P. E., and Menter, F. R., 2009. "Sensitization of the SST turbulence model to rotation and curvature by applying the Spalart-Shur correction term". ASME J. Turbomach., 131(10), p. 041010.

[10] Liu, Y., Yan, H., Liu, Y., Lu, L., and Li, Q., 2016. "Numerical study of corner separation in a linear compressor cascade using various turbulence models". Chinese J. Aeronaut., 29(3), pp. 639-652.

[11] Scillitoe, A. D., Tucker, P. G., and Adami, P., 2015. "Evaluation of RANS and ZDES methods for the prediction of three-dimensional separation in axial flow compressors". ASME Paper No. GT2015-43975.

[12] Li, W., and Liu, Y., 2019. "Evaluation of Spalart-Allmaras model with various modifications for predicting corner separation in a compressor cascade". GPPS Paper No. GPPS-BJ-2019-0177.

[13] Liu, Y., Yu, X., and Liu, B., 2008. "Turbulence models assessment for large-scale tip vortices in an axial compressor rotor". J. Propuls. Power, 24(1), pp. 15-25.

[14] Liu, B., An, G., and Yu, X., 2015. "Assessment of curvature correction and reattachment modification into the shear stress transport model within the subsonic axial compressor simulations". Proc. Inst. Mech. Eng. Part A J. Power Energy, 229(8), pp. 910-927.

[15] Spalart, P. R., 2000. "Strategies for turbulence modelling and simulations". Int. J. Heat Fluid Flow, 21(3), pp. 252-263.

[16] Shur, M. L., Strelets, M. K., Travin, A. K., and Spalart, P. R., 2000. "Turbulence modeling in rotating and curved channels: Assessing the Spalart-Shur correction". AIAA J., 38(5), pp. 784-792.

[17] Rumsey, C. L. Turbulence Modeling Resource. https://turbmodels.larc.nasa.gov/.

[18] Liu, Y., Lu, L., Fang, L., and Gao, F., 2011. "Modification of Spalart-Allmaras model with consideration of turbulence energy backscatter using velocity helicity". Phys. Lett. A, 375(24), pp. 2377-2381.

[19] Ma, L., Lu, L., Fang, J., and Wang, Q., 2014. “A study on turbulence transportation and modification of Spalart-Allmaras model for shock-wave/turbulent boundary layer interaction flow". Chinese J. Aeronaut., 27(2), pp. 200-209.

[20] Lee, K. B., Wilson, M., and Vahdati, M., 2018. "Validation of a numerical model for predicting stalled flows in a low-speed fan-part I: Modification of Spalart-Allmaras turbulence model". ASME J. Turbomach., 140(5), p. 051008.

[21] Lee, K. B., Dodds, J., Wilson, M., and Vahdati, M., 2018. "Validation of a numerical model for predicting stalled flows in a low-speed fan-part II: Unsteady analysis". ASME J. Turbomach., 140(5), p. 051009.

[22] Wilcox, D. C., 1998. Turbulence modeling for CFD. DCW industries La Canada, CA.

[23] Spalart, P. R., 2015. "Philosophies and fallacies in turbulence modeling". Prog. Aerosp. Sci., 74, pp. 1-15.

[24] Jespersen, D. C., Pulliam, T. H., and Childs, M. L., 2016. OVERFLOW turbulence modeling resource validation results. Tech. rep., NASA Ames Research Center. NAS-2016-01.

[25] Strazisar, A. J., Wood, J. R., Hathaway, M. D., and Suder, K. L., 1989. Laser anemometer measurements in a transonic axial-flow fan rotor. Tech. rep., NASA Lewis Research Center, Cleveland, Ohis, USA. NASA-TP-2879.

[26] Grosvenor, A. D., 2007. "RANS prediction of transonic compressive rotor performance near stall". ASME Paper No. GT2007-27691.

[27] Fidalgo, J. V., Hall, C. A., and Colin, Y., 2012. “A study of fan-distortion interaction within the NASA Rotor 67 transonic stage". ASME J. Turbomach., 134(9), p. 051011.

[28] Cumpsty, N., and Horlock, J., 2006. "Averaging nonuniform flow for a purpose". ASME J. Turbomach., 128(1), pp. 120-129.

[29] Spalart, P. R., and Rumsey, C. L., 2007. "Effective inflow conditions for turbulence models in aerodynamic calculations". AIAA J., 45(10), pp. 2544-2553.

[30] Vahdati, M., Sayma, A. I., Freeman, C., and Imregun, M., 2005. "On the use of atmospheric boundary conditions for axial-flow compressor stall simulations". ASME J. Turbomach., 127(2), pp. 349-351.

[31] Pope, S., 2000. Turbulent Flows. Cambridge University Press, Cambridge, UK.

[32] Im, H., Chen, X. Y., and Zha, G., 2012. "Detached-eddy simulation of rotating stall inception for a full-annulus transonic rotor". J. Propuls. Power, 28(4), pp. $782-$ 
798.

[33] Schlichting, H., and Gersten, K., 2016. Boundary-layer theory. Springer.

[34] Vo, H. D., Tan, C. S., and Greitzer, E. M., 2008. "Criteria for spike initiated rotating stall". ASME J. Turbomach., 130(1), p. 011023.

[35] Hewkin-Smith, M., Pullan, G., Grimshaw, S. D., Greitzer, E. M., and Spakovszky, Z. S., 2019. "The role of tip leakage flow in spike-type rotating stall inception". ASME J. Turbomach., 141(6), p. 061010. 